\title{
Polypharmacy and excessive dosing: psychiatrists' perceptions of antipsychotic drug prescription
}

\author{
HIROTO ITO, ASUKA KOYAMA and TERUHIKO HIGUCHI
}

\begin{abstract}
Background Despite extensive research and recommendations regarding the optimal prescription of antipsychotic drugs, polypharmacy and excessive dosing still prevail.
\end{abstract}

Aims To identify the factors associated with the polypharmacy and excessive dosing phenomena.

Method We studied 139 patients with schizophrenia, in 19 acute psychiatric units in Japanese hospitals, who were due to be discharged between October and December 2003.We examined patient characteristics, nurses' requests, and psychiatrists' characteristics and perceptions of prescribing practice and algorithms.

Results Polypharmacy and excessive dosing were observed in 96 cases. Logistic regression analysis revealed that the use of multiple medications and excessive dosing were influenced by the psychiatrist's scepticism towards the use of algorithms, nurses' requests for more drugs and the patient's clinical condition.

Conclusions Educational interventions are necessary for psychiatrists and nurses to follow evidence-based guidelines or algorithms.

Declaration of interest None.
Polypharmacy involves the concomitant administration of two or more drugs. Excessive dosing refers to doses greater than optimal daily dosage of between 300 and $1000 \mathrm{mg}$ of chlorpromazine equivalent (Lehman \& Steinwachs, 1998). Despite extensive research and recommendations as to the optimal prescription of antipsychotics, polypharmacy and excessive dosing are still widely prevalent in clinical practice in Canada (Procyshyn et al, 2001), East Asia (Bitter et al, 2003; Chong et al, 2004) and the USA (Diaz \& de Leon, 2002; Bitter et al, 2003; Sohler et al, 2003). Polypharmacy is strongly associated with excessive dosing (Lelliott et al, 2002). Although several causes of polypharmacy and excessive dosing have been proposed, few studies have explored psychiatrists' perceptions of prescribing practice since the establishment of Benson's conceptual approach as a three-stage decision-making process: the psychiatrist's decision to prescribe any psychopharmaceutical, the decision to prescribe an antipsychotic drug and the determination of antipsychotic drug dosage (Benson, 1983). In this study, we aimed to identify the factors associated with the polypharmacy and excessive dosing phenomena. We examined patient characteristics, nurses' requests for drugs, the characteristics of the prescribing psychiatrists and their perceptions of prescribing practices and algorithms in Japan.

\section{METHOD}

\section{Participants}

We invited all public and private hospitals with acute psychiatric care units (as defined by the Japanese reimbursement system) to participate in the study. Acute psychiatric care units under this reimbursement system have strict criteria: the hospitals have to participate in the regional psychiatric emergency system; the levels of staffing are more than twice those of general psychiatric units; at least one seclusion room should be available; more than $40 \%$ of patients come from the community; and the patients should be discharged within the shortest possible period. A total of 19 hospitals (3 public and 16 private) agreed to participate in the study. There were no significant differences in the characteristics of these hospitals, such as size, ownership and the number of beds.

All the patients with schizophrenia discharged from the participating units between 1 October and 25 December 2003 were invited to take part in the study. Of 251 patients, $179(71.3 \%)$ agreed to participate and provided written informed consent, a sample size considered to be sufficient to give an overview of the prescribing patterns during the study period. Thirty-four patients were eliminated from the analysis because of missing data, and a further six patients were eliminated because they had not been prescribed antipsychotics. Thus, we used data from 139 patients for our analysis. There was no significant difference in the age and gender of the patients selected for inclusion and exclusion.

The study was approved by the institutional review board of the Japanese National Centre of Neurology and Psychiatry and also by the institutional review board or board of directors of each participating hospital. Research coordinators collected patient information from the participating hospitals without identifying the patients.

\section{Patient characteristics}

We defined a standard dosage group comprising patients who were receiving one antipsychotic drug with a dosage of less than $1000 \mathrm{mg}$ chlorpromazine equivalent. The remaining patients constituted the non-standard dosage group. We asked psychiatrists about the clinical variables of the patients, including psychiatric diagnosis and length of illness. All the patients had a clinical diagnosis of schizophrenia based on DSM-IV criteria (American Psychiatric Association, 1994). Psychiatrists also rated the patients on the Global Assessment of Functioning (GAF; American Psychiatric Association, 1994) scale both at admission and at discharge. Lower GAF scores indicate greater disability. Nurses provided patient demographic variables and reported the use of seclusion and physical restraint during in-patient care. 


\section{Psychiatrist characteristics and prescribing perceptions}

We asked the 78 psychiatrists treating the 139 patients to provide information on their demographic variables (age and gender), medical qualifications, length of clinical experience, and perceptions of prescribing practice and dosing algorithms.

The psychiatrists were asked to describe their perceptions of prescribing practice and algorithms before the patients were recruited. Questions on prescribing practice included cost considerations, familiarity with the research literature and the importance of 'experience-based' prescribing. Perceptions of algorithms were elicited by questions such as 'I understand the contents of an algorithm', 'An algorithm disregards individual patient characteristics', 'I doubt the validity and evidence of an algorithm' and 'I think that an algorithm is necessary for clinical practice'. Each item was rated using a four-point Likert scale (1, strongly disagree; 2 , disagree; 3 , agree; 4 , strongly agree). Japanese translations of algorithms and guidelines used in the UK (Taylor et $a l, 2001$ ) and the USA (American Psychiatric Association, 1997) were available to these psychiatrists in addition to algorithms developed in Japan.

\section{Nurses' requests for drugs}

The nurses completed a questionnaire survey. The questionnaire asked whether they believed that it was necessary to increase the current dosage of medication or add another drug; to decrease the current dosage or number of drugs; or to change the current drug. We also asked the nurses to indicate the reason why they believed a change was necessary in each case.

\section{Statistical analysis}

All dosages of antipsychotic drugs were converted into chlorpromazine equivalents to facilitate comparisons (BezchlibnykButler \& Jeffries, 1998; Inagaki et al, 1999). We used $t$-tests to compare mean scores and chi-squared tests to compare categorical data. The Mann-Whitney test was used to compare the rank data between the standard and non-standard dosage groups. Multiple logistic regression analysis was used to assess the independent and interactive effects of the multiple factors that could contribute to prescribing practice. After we examined the relationship of each variable in the two prescribing practice groups, we included only the significant variables when comparing the two groups in the logistic regression analysis. All tests were two-tailed. Analyses were performed using the Statistical Package for the Social Sciences, version 11.0.

\section{RESULTS}

Table 1 shows the patterns of prescription of antipsychotic drugs. There were 37 patients $(27 \%)$ in the standard dosage group: 29 of the 37 were taking atypical antipsychotics. Of the 102 patients $(73 \%)$ in the non-standard dosage group, 96 were taking more than one drug, 32 of whom were also prescribed excessive dosages. In the non-standard dosage group, 57 patients were given both typical and atypical antipsychotics simultaneously.

The psychiatrists' mean age was 41.3 years $\quad($ s.d. $=10.7)$, with 12.9 years (s.d.=10.8) experience in psychiatric services. Of the 78 psychiatrists, $50(64 \%)$ were designated psychiatrists with extra training; these individuals were qualified to make the decision for compulsory admission under the Mental Health and Welfare Law of Japan 1995. Regarding the psychiatrists' demographic variables, medical qualifications, length of clinical experience and perceptions of prescribing practice, no significant difference was observed between the standard and non-standard dosage groups. There were, however, significant differences in the psychiatrists' perceptions of algorithms. Psychiatrists caring for patients in the non-standard dosage group were significantly more likely to agree with the statement 'I doubt the validity and evidence of an algorithm' $(z=-2.95$, $P=0.003$ ) and more likely to disagree with the statement 'I think that an algorithm is necessary for clinical practice' $(z=-2.49$, $P=0.013)$ compared with those in the standard dosage group.

Patient characteristics are shown in Table 2. There was no significant difference in age or gender between the standard and non-standard dosage groups. The nonstandard dosage group had a significantly longer duration of illness than the standard dosage group. There was no significant difference in involuntary admission or the use of physical restraint during in-patient care. The GAF scores at admission did not differ significantly, whereas the GAF score of the non-standard dosage group at discharge was significantly lower than that of the standard dosage group.

Forty-nine $(59 \%)$ of the 83 nurses caring for our 139 patients were men. The nurses' mean age was 35.3 years (s.d.=9.3), and they had an average of 9.4 years' (s.d.=7.3) experience in psychiatric services. Nurses endorsed the statement that 'I would like to ask a psychiatrist to increase the current dosage or add another drug' for 39 patients. The proportion of nurses agreeing with this statement was significantly greater in the non-standard

Table I Prescription of antipsychotic medication to the study participants

\begin{tabular}{|c|c|c|c|}
\hline & \multicolumn{2}{|c|}{ Dosage } & \multirow{2}{*}{$\begin{array}{l}\text { Total } \\
n(\%)\end{array}$} \\
\hline & $\begin{array}{c}<1000 \mathrm{mg} \text { CPZeq } \\
n(\%)\end{array}$ & $\begin{array}{c}1000 \mathrm{mg} \text { CPZeq and over } \\
n(\%)\end{array}$ & \\
\hline \multicolumn{4}{|c|}{ Standard dosage group $(n=37)$} \\
\hline \multicolumn{4}{|l|}{ Monotherapy } \\
\hline Typical & $8 \quad(5.8)$ & & $8 \quad(5.8)$ \\
\hline Atypical & $29(20.9)$ & & $29(20.9)$ \\
\hline \multicolumn{4}{|c|}{ Non-standard dosage group $(n=102)$} \\
\hline \multicolumn{4}{|l|}{ Monotherapy } \\
\hline Typical & & $2(1.4)$ & $2(1.4)$ \\
\hline Atypical & & $4(2.9)$ & $4 \quad(2.9)$ \\
\hline \multicolumn{4}{|l|}{ Polypharmacy } \\
\hline Typical+typical & $22(15.8)$ & $10(7.2)$ & $32(23.0)$ \\
\hline Typical+atypical & $35(25.2)$ & $22(15.8)$ & $57(41.0)$ \\
\hline Atypical+atypical & $7 \quad(5.0)$ & $0(0.0)$ & $7 \quad(5.0)$ \\
\hline Total & $101 \quad(72.7)$ & $38(27.3)$ & $139(100.0)$ \\
\hline
\end{tabular}

CPZeq; chlorpromazine equivalent. 
dosage group than in the standard dosage group. The reasons nurses requested a change in treatment included 'no improvement in symptoms' (24 patients; 62\%), 'deterioration in symptoms' (9 patients; $23 \%$ ), 'beyond nursing care' (4 patients; $10 \%$ ) and 'other' ( 2 patients, $5 \%$ ). There was no significant difference between the standard and non-standard dosage groups with regard to the reasons for the desired alteration in drug treatment.

Logistic regression analysis revealed that the non-standard dosage group was significantly more likely to have both a longer duration of illness and a lower level of functioning as evaluated by the GAF scale (Table 3 ). The analysis also showed that the psychiatrists' perceptions of algorithms were associated with polypharmacy and excessive dosing. Nurses in the nonstandard dosage group were more likely to believe that their patients needed more drugs than those in the standard dosage group.

\section{DISCUSSION}

Antipsychotic polypharmacy and excessive dosing continue to be used for the treatment of schizophrenia in acute psychiatric care units despite current recommendations. The results indicate that polypharmacy and excessive dosing are associated with both psychiatrists' perceptions of the use of algorithms and nurses' requests for more drugs, as well as the clinical variables of the patients.

\section{Methodological considerations}

We examined the factors influencing the patterns of prescription of antipsychotics using three explanatory variables: patient characteristics, nurses' requests for drugs and psychiatrists' perceptions of best prescribing practice and algorithms. The psychiatrists' perceptions were subjective measures and we did not conduct an objective assessment of this variable. Also, we were not able to examine subjective patient outcomes, such as satisfaction with medication and quality of life, although the psychiatrists rated the patients' level of functioning using the GAF score. Ideally, one should examine the relationship between prescribing patterns and the longterm outcomes of patients.

Every acute psychiatric care unit had the same staffing ratio of patients to nurses. The size and ownership of the hospitals did

Table 2 Patient characteristics and nurses' requests for drugs

Standard dosage Non-standard dosage Test statistic $P$

group $(n=37) \quad$ group $(n=102)$

\begin{tabular}{|c|c|c|c|c|}
\hline \multicolumn{5}{|l|}{ Patient characteristics } \\
\hline Age, years: mean (s.d.) & $38.7(13.5)$ & $41.2(13.5)$ & 0.971 & 0.33 \\
\hline \multicolumn{5}{|l|}{ Gender, $n(\%)$} \\
\hline Male & $22(60)$ & $69(68)$ & $0.81^{2}$ & 0.42 \\
\hline Female & $15 \quad(40)$ & $33 \quad(32)$ & & \\
\hline Length of illness, years: mean (s.d.) & $9.5(10.2)$ & $16.5(11.8)$ & $3.23^{\prime}$ & $<0.01$ \\
\hline \multicolumn{5}{|l|}{ Involuntary admission, $n$ (\%)' } \\
\hline Yes & $24 \quad(65)$ & $69(68)$ & $0.09^{2}$ & 0.84 \\
\hline No & $13 \quad(35)$ & $33 \quad(32)$ & & \\
\hline \multicolumn{5}{|l|}{$\begin{array}{l}\text { Restrction during in-patient care, } \\
n(\%)\end{array}$} \\
\hline Yes & 8 (22) & $15 \quad(15)$ & $0.94^{2}$ & 0.44 \\
\hline No & $29(78)$ & $87 \quad(85)$ & & \\
\hline \multicolumn{5}{|l|}{ GAF score: mean (s.d.) } \\
\hline GAF at admission & $28.3(14.9)$ & $31.8(14.1)$ & 1.28 & 0.20 \\
\hline GAF at discharge & $64.8(16.2)$ & $57.2(14.5)$ & $2.66^{1}$ & $<0.01$ \\
\hline \multicolumn{5}{|l|}{$\begin{array}{l}\text { Nurses' requests for drugs: } \\
\text { mean score (s.d.) }\end{array}$} \\
\hline $\begin{array}{l}\text { I would like to ask a psychiatrist to } \\
\text { increase the current dosage or add } \\
\text { another drug }\end{array}$ & I.4 (0.8) & I.8 (0.9) & $2.55^{\prime}$ & 0.01 \\
\hline $\begin{array}{l}\text { I would like to ask a psychiatrist to } \\
\text { decrease the current dosage or } \\
\text { number of drugs }\end{array}$ & I.4 (0.7) & $1.4(0.8)$ & $0.38^{\prime}$ & 0.71 \\
\hline $\begin{array}{l}\text { I would like to ask a psychiatrist to } \\
\text { change the current drug }\end{array}$ & $1.5(0.8)$ & $1.8(0.9)$ & $\mathrm{I} .5 \mathrm{I}^{1}$ & 0.13 \\
\hline
\end{tabular}

GAF, Global Assessment of Functioning.

I. Independent $t$-test.

2. Chi-squared test.

3. Rated as I, strongly disagree; 2 , disagree; 3 , agree; 4 , strongly agree.

Table 3 Logistic regression results predicting standard and non-standard dosage groups

\begin{tabular}{|c|c|c|c|}
\hline & Adjusted odds ratio & $95 \% \mathrm{Cl}$ & $P$ \\
\hline \multicolumn{4}{|l|}{ Patient characteristics } \\
\hline Length of illness & 1.05 & $1.0 \mathrm{I}-\mathrm{I} .10$ & 0.02 \\
\hline GAF score at discharge & 0.98 & $0.95-1.01$ & 0.20 \\
\hline \multicolumn{4}{|l|}{ Psychiatrist's perceptions ${ }^{2}$} \\
\hline I doubt the validity and evidence of an algorithm & 2.86 & $\mathrm{I} .02-8.0 \mathrm{I}$ & 0.046 \\
\hline I think that an algorithm is necessary for clinical practice & 0.55 & $0.25-1.21$ & 0.14 \\
\hline \multicolumn{4}{|l|}{ Nurses' requests ${ }^{2}$} \\
\hline $\begin{array}{l}\text { I would like to ask a psychiatrist to increase the current } \\
\text { dosage or add another drug }\end{array}$ & 1.76 & $1.05-2.93$ & 0.03 \\
\hline
\end{tabular}

I. Standard dosage group, 0 ; non-standard dosage group, I.

2. Rated as I, strongly disagree; 2 , disagree; 3 , agree; 4 , strongly agree.

not differ between the standard and nonstandard dosage groups; however, we did not examine additional institutional characteristics and staffing, owing to substantial missing and inappropriate data for analysis. This study was not a retrospective review of patient records; rather, we obtained prospective data at the point when 
discharge was planned. Furthermore, the patient, nurse and psychiatrist data were collected separately and matched later. Thus, we were able to analyse prescribing patterns for individual patients rather than using a group analysis.

The number of participating hospitals was small because we used strict recruitment criteria. In Japan, there are still many psychiatric care units that are similar to rehabilitation units in Western countries. As Japan is now in a transitional period from long-term to acute hospital care, various measures are employed to shorten the patients' length of stay. One such measure is that an acute psychiatric care unit is strictly defined in the reimbursement system. We used this criterion to select our hospital sample; however, only a limited number of hospitals have been officially designated as acute psychiatric care units. Therefore, our sample might not be nationally representative of all hospitals in Japan with acute psychiatric care units. To reduce the burden on participating hospitals the study period was only 2 months, and because of this the number of patients who met the diagnostic criteria during that period was limited.

\section{Benefits and risks of combination therapy}

It is not appropriate that polypharmacy and high-dosage prescribing should always be viewed as a poor prescribing pattern, because using more than one antipsychotic drug can be effective in some patients, and different antipsychotics have different effects on different symptoms of psychosis (Taylor, 2002). The Royal College of Psychiatrists' consensus statement in the UK (Royal College of Psychiatrists, 1993) suggests that there are some justifiable cases of temporary polypharmacy, including making a gradual change from one drug to another (Thompson, 1994). Although sulpiride augmentation of clozapine is suggested to be of benefit by a randomised controlled trial (Shiloh et al, 1997), evidence for the efficacy of combining antipsychotics is limited (Freudenreich \& Goff, 2002). There are potential adverse effects, some of which are even life-threatening (Centorrino et al, 2004). Polypharmacy is associated with early death (Waddington et al, 1998). Reilly et al (2000) reported that use of thioridazine was a predictor of $\mathrm{QT}_{c}$ prolongation, and Ray et al (2001) suggested that even moderate doses of antipsychotics would increase the risk of sudden cardiac death. Asian patients are more vulnerable to side-effects and might require less antipsychotic medication than European patients (Ungvari et al, 1996; Chong et al, 2004).

Despite these known risks, polypharmacy and excessive dosing with antipsychotics persist in Japan. An inadequate knowledge of pharmacology may underlie this phenomenon (Kingsbury et al, 2001; Procyshyn et al, 2001). Based on a questionnaire regarding the use of depot formulations, Patel et al (2003) suggested that psychiatrists' knowledge about maintenance medication was positively associated with attitudes toward the medication.

Concurrent prescription of atypical and typical antipsychotics is not recommended in principle by the National Institute for Clinical Excellence in the UK (National Institute for Clinical Excellence, 2002). It rarely improved outcomes, while it increased use of anticholinergic medication (Taylor et al, 2000). In our study, we found the combination of typical and atypical antipsychotics to be a popular prescribing pattern. Four atypical antipsychotics are available in Japan, including risperidone (since 1996), perospirone, quetiapine and olanzapine (since 2001), but clozapine has not been approved yet. The results suggest that many psychiatrists do not fully understand the mechanisms and advantages of atypical antipsychotics, and do not want to change their prescribing patterns.

\section{Implications}

There is much speculation about the factors associated with polypharmacy and excessive dosing. Previous studies suggest that these might include treatment setting; patient factors, such as age, severity of illness and length of illness (Benson, 1983; Remington et al, 2001; Bitter et al, 2003; Sohler et al, 2003; Centorrino et al, 2004); and the provider's knowledge of pharmacology, the local prescribing culture, personal experience and familiarity with the research literature (Benson, 1983; Kingsbury et al, 2001; Procyshyn et al, 2001). However, few of these factors have been proved to be associated with patient treatment. These results are consistent with other observations with regard to the severity (Sohler et al, 2003) and chronicity (Benson, 1983; Diaz \& de Leon, 2002) of patients' illness.

As in the investigation by Harrington et al (2002) of the issue of medication given at the discretion of nurses, most nurses requested higher doses of medication for the reason of patient symptoms in our study $(85 \%)$. The process of psychiatrists' agreement is unknown; however, there are two possibilities: one is that a patient still has a psychosis, and the other is that they wish to control patient behaviour. Scepticism towards algorithms and scientific evidence still exists among psychiatrists, which leads to their relying solely on clinical experience when prescribing antipsychotic medication. Consequently, psychiatrists who are sceptical about algorithms are potential targets for educational intervention. Also, educational programmes detailing scientific advances can be effective for healthcare providers, including psychiatrists and nurses.

\section{Future interventions}

Education, guidelines and algorithms are mentioned in the research literature as ways to avoid irrational polypharmacy and high doses for the purpose of unnecessary sedation (Ungvari et al, 1997; Lehman \& Steinwachs, 1998; Covell et al, 2002). In fact, the introduction of educational programmes and guidelines is reportedly effective (Avorn et al, 1992; Grimshaw \& Russell, 1993), but it also was reported that the degree of performance improvement varied (Grimshaw \& Russell, 1993) and that systematic practice-based interventions and outreach visits were necessary (Davis $e t$ al, 1995). McCue et al (2003) suggested that a rational strategy for prescribing can lead to a decrease in adverse drug reactions and an improvement in patient outcomes, even when using more than one antipsychotic drug.

We did not examine the effects of educational intervention in this study. An intervention study is necessary to assess the feasibility and impact of implementing an evidence-based medication algorithm; we plan to include this in our next research protocol.

\section{ACKNOWLEDGEMENT}

The study was supported in part by the Ministry of Health, Labour and Welfare, Japan. The opinions expressed in this article are those of the authors and do not represent the official views of the Ministry.

\section{REFERENCES}

American Psychiatric Association (1994) Diagnostic and Statistical Manual of Mental Disorders (4th edn) (DSM-IV).Washington, DC: APA 
American Psychiatric Association (1997) Practice guidelines for the treatment of patients with schizophrenia. American Journal of Psychiatry, 154 (suppl.), $1-63$.

Avorn, J., Soumerai, S. B., Everitt, D. E., et al (1992) A randomized trial of a program to reduce the use of psychoactive drugs in nursing homes. New England Journal of Medicine, 327, 168-173.

Benson, P. R. (1983) Factors associated with antipsychotic drug prescribing by southern psychiatrists. Medical Care, 2I, 639-654.

Bezchlibnyk-Butler, K. Z. \& Jeffries, J. J. (1998) Clinical Handbook of Psychotropic Drugs (8th edn). Seattle,WA: Hogrefe \& Huber.

Bitter, I., Chou, J. C., Ungvari, G. S., et al (2003) Prescribing for inpatients with schizophrenia: an international multi-center comparative study. Pharmacopsychiatry, 36, I43-149.

Centorrino, F., Goren, J. L., Hennen, J., et al (2004) Multiple versus single antipsychotic agents for hospitalized psychiatric patients: case-control study of risks versus benefits. American Journal of Psychiatry, 16I 700-706.

Chong, M. Y., Tan, C. H., Fujii, S., et al (2004) Antipsychotic drug prescription for schizophrenia in East Asia: rationale for change. Psychiatry and Clinical Neurosciences, 58, 61-67.

Covell, N. H., Jackson, C. T., Evans, A. C., et al (2002) Antipsychotic prescribing practice in Connecticut's pubic mental health system: rates of changing medications and prescribing styles. Schizophrenia Bulletin, 28, 17-29.

Davis, D. A., Thomson, M. A., Oxman, A. D., et al (1995) Changing physician performance: a systematic review of the effect of continuing medical education strategies. JAMA, 274, 700-705

Diaz, F. J. \& de Leon, J. (2002) Excessive antipsychotic dosing in two US state hospitals. Journal of Clinical Psychiatry, 63, 998-1003.

Freudenreich, O. \& Goff, D. C. (2002) Antipsychotic combination therapy in schizophrenia: a review of efficacy and risks of current combinations. Acto Psychiatrica Scandinavica, 106, 323-330.

Grimshaw, J. M. \& Russell, I.T. (1993) Effect of clinical guidelines on medical practice: a systematic review of rigorous evaluations. Lancet, 342, 1317-1322.

Harrington, M., Lelliott, P., Paton, C., et al (2002) The results of a multi-centre audit of the prescribing of antipsychotic drugs for in-patients in the UK. Psychiatric Bulletin, 26, 4I4-4I8.

Inagaki, A., Inada, T., Fujii, Y., et al (1999) Equivalent Dose of Psychotropics (in Japanese). Tokyo: Seiwa Shoten

\section{Kingsbury, S. J., Yi, D. \& Simpson, G. M. (200I)}

Rational and irrational polypharmacy. Psychiatric

Services, 52, 1033-1036.

Lehman, A. F. \& Steinwachs, D. M. (1998)

Translating research into practice: the Schizophrenia Patients Outcomes Research Team (PORT)

treatment recommendations. Schizophrenia Bulletin, 24 $1-10$.

Lelliott, P., Paton, C., Harrington, M., et al (2002)

The influence of patient variables on polypharmacy and combined high dose of antipsychotic drugs prescribed for in-patients. Psychiatric Bulletin, 26, 4II-4I4.

McCue, R. E., Waheed, R. \& Urcuyo, L. (2003)

Polypharmacy in patients with schizophrenia. Journal of Clinical Psychiatry, 64, 984-989.

National Institute for Clinical Excellence (2002) Guidance on the Use of Newer (Atypical) Antipsychotic

\section{CLINICAL IMPLICATIONS}

- Prescribing patterns that include antipsychotic polypharmacy and excessive dosing persist in clinical practice in Japan.

- Major associated factors were psychiatrists' perceptions of medication algorithms and nurses' requests for more drugs, as well as the length of illness and level of functioning of patients.

- Educational interventions are necessary for psychiatrists and nurses to follow evidence-based guidelines or algorithms.

\section{LIMITATIONS}

- The numbers of participating hospitals and patients were limited owing to strict inclusion criteria and a short research period.

- Subjective patient outcomes were not examined.

- There was no intervention to improve prescribing practice.

HIROTO ITO, PhD, National Institute of Mental Health, Tokyo; ASUKA KOYAMA, MSc, Department of Mental Health, University of Tokyo; TERUHIKO HIGUCHI, MD, PhD, National Centre of Neurology and Psychiatry, Musashi Hospital, Tokyo, Japan

Correspondence: Dr Hiroto Ito, National Institute of Mental Health, 4-I-I Ogawa-Higashi, Kodaira, Tokyo 187-8502, Japan. E-mail: Hiroto0405@aol.com

(First received 16 June 2004, final revision 16 November 2004, accepted 20 November 2004)

Drugs for The treatment of Schizophrenia. Technology Appraisal Guidance No. 43. London: NICE.

Patel, M. X., Nikolaou, V. \& David, A. S. (2003) Psychiatrists' attitudes to maintenance medication for patients with schizophrenia. Psychological Medicine, 33, 83-89.

Procyshyn, R. M., Kennedy, N. B., Tse, G., et al (200I) Antipsychotic polypharmacy: a survey of discharge prescriptions from a tertiary care psychiatry institution Canadian Journal of Psychiatry, 46, 334-339.

Ray, W. A., Meredith, S., Thapa, P. B., et al (200I) Antipsychotics and the risk of sudden cardiac death. Archives of General Psychiatry, 58, |161-1167.

Reilly, J. G., Avis, S. A., Ferrier, I. N., et al (2000) QT -interval abnormalities and psychotropic drug therapy in psychiatric patients. Lancet, 355, 1048-1052.

Remington, G., Shammi, C. M., Sethna, R., et al (200I) Antipsychotic dosing patterns for schizophrenia in three treatment settings. Psychiatric Services, 52 $96-98$.

Royal College of Psychiatrists (1993) Consensus Statement on the Use of High Dose Antipsychotic Medication. Council Report CR26. London: Royal College of Psychiatrists.

Shiloh, R., Zemishlany, Z., Aizenberg, D., et al (1997) Sulpiride augmentation in people with schizophrenia partially responsive to clozapine. A double-blind, placebo-controlled study. British Journal of Psychiatry, I7I, 569-573.

Sohler, N. L., Walkup, J., McAlpine, D., et al (2003) Antipsychotic dosage at hospital discharge and outcomes among persons with schizophrenia. Psychiatric Services, 54, $1258-1263$.
Taylor, D. (2002) Antipsychotic prescribing - time to review practice. Psychiatric Bulletin, 26, 40I-402.

Taylor, D., Mace, S., Mir, S., et al (2000) A prescription survey of the use of atypical antipsychotics for hospital inpatients in the United Kingdom. International Journal of Psychiatry in Clinical Practice, 4 $41-46$.

Taylor, D., McConnell, D., McConnell, H., et al (200I) The Maudsley 2001 Prescribing Guidelines (6th edn). London: Taylor \& Francis.

Taylor, D., Mir, S., Mace, S., et al (2002) Coprescribing of atypical and typical antipsychotics prescribing sequence and documented outcome. Psychiatric Bulletin, 26, 170-172.

Thompson, C. (1994) The use of high-dose antipsychotic medication. British Journal of Psychiatry I64, 448-458.

Ungvari, G. S., Pang, A. H. T., Chiu, H. F. K., et al (1996) Psychotropic drug prescription in rehabilitation: a survey in Hong Kong. Social Psychiatry and Psychiatric Epidemiology, 3I, 288-291.

Ungvari, G. S., Chow, L. Y., Chiu, H. F., et al (1997) Modifying psychotropic drug prescription patterns: a follow-up survey. Psychiatry and Clinical Neurosciences, $\mathbf{5 I}$ 309-314.

Waddington, J. L., Youssef, H. A. \& Kinsella, A. (1998) Mortality in schizophrenia. Antipsychotic polypharmacy and absence of adjunctive anticholinergics over the course of a 10-year prospective study. British Journal of Psychiatry, I73, 325-329. 